\title{
A Prática do Yoga no Pré-natal: redução do estresse e outros achados
}

\author{
The Prenatal Yoga Practice: \\ stress reduction and other findings
}

\section{Maria Antonieta Nascimento Araújo' Beatriz Gavazzi Lopes Prado²}

\author{
'Escola Bahiana de Medicina e Saúde Pública (Salvador). Bahia, Brasil. antonieta.araujo@bahiana.edu.br \\ ${ }^{2}$ Autora para correspondência. Escola Bahiana de Medicina e Saúde Pública (Salvador). Bahia, Brasil. beatrizprado16.1@bahiana.edu.br
}

RESUMO | Na gestação, a mulher está suscetivel a diversos fatores que geram estresse recorrente. Tais fatores, descritos como estressores, são considerados disparadores de sintomas de angústia que, eventualmente, evoluem para quadros de ansiedade e depressão (pré e pós-parto), e mesmo respostas orgânicas, como a elevação das taxas de biomarcadores do estresse como o cortisol, o de maior abundância. O yoga foi apontado como benéfico para o sistema hipotálamo-pituitária-adrenal (HPA), repercutindo no Sistema Nervoso Autônomo (SNA), com consequente melhora na saúde física e mental. Assim, o objetivo deste estudo foi identificar e sistematizar o conhecimento produzido pela literatura científica acerca dos efeitos do yoga sobre o estresse em mulheres grávidas. Para tanto, foi desenvolvida uma revisão integrativa de conteúdos, em artigos da base de dados do Sistema Online de Busca e Análise de Literatura Médica (MEDLINE), no período de 2012 a 2019. Mediante a busca em descritores - yoga; gravidez; pregnancy; stress - chegou-se a 124 estudos, entre os quais, 13 deles obedeciam aos critérios de inclusão para serem revisados. Depois das análises, os desfechos das intervenções com yoga foram categorizados enquanto efeitos primários e secundários, os primeiros refletindo os sintomas do estresse na gestação e os secundários representando os seus efeitos no que tange a dor e desconforto na gestação e parto e marcadores de saúde do recém-nascido. Conclusão: o yoga no pré-natal de gestantes foi considerado eficaz e os benefícios levantados colocam-na como uma estratégia que pode ser prescrita, tanto de forma preventiva como complementar no tratamento dos sintomas do estresse na gestação. Acrescenta-se que, tendo em vista os impactos psicológicos do surto pandêmico do Covid-19, na atualidade, a prática de yoga pode ser uma importante ferramenta para o cuidado da saúde mental de gestantes neste período.

PALAVRAS-CHAVE: Yoga. Stress psicológico. Gravidez. Intervenção precoce.
ABSTRACT | During pregnancy, women are susceptible to several factors that generate recurrent stress. Such factors, described as stressors, are considered triggers of distress symptoms that eventually develop into anxiety and depression (pre and postpartum) and even organic responses, such as increased rates of stress biomarkers like cortisol, the most abundant. Yoga was indicated as beneficial to the hypothalamus-pituitary-adrenal system (HPA), affecting the Autonomic Nervous System (ANS), with consequent improvement in physical and mental health. Thereby, the aim of this study was to identify and systematize the knowledge produced by literature on the effects of yoga practice by pregnant women on prenatal stress. To this end, an integrative content review was developed in articles from the database of the Medical Literature Analysis and Retrieval System Online (MEDLINE), from 2012 to 2019. Through the search on descriptors yoga; pregnancy; pregnancy; stress - 124 studies were obtained, among which, 13 of them met the inclusion criteria to be reviewed. After the analyzes, the outcomes of the yoga interventions were categorized as primary and secondary effects, the primary reflecting the symptoms of stress during pregnancy and the secondary representing their effects with respect to pain and discomfort during pregnancy and childbirth and health markers of the newborn. Conclusion: prenatal yoga for pregnant women is considered effective and its benefits place it as an strategy that can be prescribed, both in a preventive and complementary way in the treatment of symptoms and mood disorders during pregnancy. It should be added that, in view of the psychological impacts of the Covid-19 pandemic outbreak, today, the practice of yoga can be an important tool for the mental health care of pregnant women.

KEYWORDS: Yoga. Psychological stress. Pregnancy. Early intervention. 


\section{Introdução}

O estresse foi descrito pela primeira vez, na Ciência, por Selye (1950), que o apresentou como uma síndrome, fruto da interação biológica entre os danos (produto do agente estressor) e a defesa (reações biológicas) do organismo, como uma reação desse frente à situações que vão além do seu limite de compreensão e/ou ação.

O estresse emocional está relacionado à necessidade do indivíduo de lidar com fatores externos ou internos ao organismo, que se apresentam como estressores, capazes de criar tensões, demandando-lhe respostas (SADIR, BIGNOTTO \& LIPP, 2010; SELYE, 1950).

Tais respostas ocorrem tanto em nível psicológico, gerando angústia e sofrimento psíquico (ARANTES \& VIEIRA, 2002) como fisiológico, com a ativação automática do eixo hipotálamo-pituitária-adrenal - o que interfere no funcionamento do sistema nervoso parassimpático, levando a um aumento das taxas de cortisol no sangue (HPA) (LINDSAY \& NIEMAN, 2005; MONTENEGRO et al, 2010).

Durante o período gestacional, a mulher está suscetível a diversos fatores estressores próprios dessa fase, que podem gerar sintomas recorrentes de estresse (RODRIGUES \& SCHIAVO, 2011; GLAZIER et al., 2004).

Quadros de estresse em gestantes foram considerados disparadores de sintomas de angústia que, eventualmente, evoluíram para quadros de ansiedade (GLAZIER et al., 2004; PAMPAKA et al., 2018), depressão recorrente ou crônica, tanto pré quanto pós-parto (ROMERO-GONZALES et al., 2020), assim como o risco de prejuízos no vínculo afetivo mãe bebê (GLAZIER et al., 2004; PAMPAKA et al., 2018). Há estudos que relacionam ainda o estresse ao suicídio e ao infanticídio em gestantes adolescentes (FIELD et al., 2013b; FIELD et al., 2012).

A ansiedade e depressão são resposta emocionais recorrentes neste período gestacional, e tendem a afetar negativamente a progressão saudável da gestação (SATYAPRIYA et al, 2013). A depressão, tanto pré quanto pós-natal é uma das complicações mais comuns no tocante à este período, afetando até $60.8 \%$ das gestantes em algumas populações (PAMPKA et al, 2018). Este quadro pode se fazer presente a qualquer momento no decorrer de um ano da gestação e sua duração varia de mulher para mulher (PAMPKA et al, 2018). Em relação a ansiedade materna pré-natal, a mesma é muitas vezes menosprezada devido a dificuldade de se conceituar a sua representação, bem como pela dificuldade de avalia-la (NEWHAM et al, 2014). Esta negligência, por sua vez, pode desencadear uma intensificação dos seus efeitos adversos, como disparador para demais complicações, dentre elas, a depressão (NEWHAM et al, 2014).

Alguns fatores específicos de estresse podem ser listados neste período gravídico, como o medo em relação ao parto e à própria morte ou do bebê, o receio das mudanças que ocorrerão com a maternidade, preocupações financeiras, dificuldade nos relacionamentos, entre outros, (RODRIGUES \& SCHIAVO, 2011; GLAZIER et al., 2004).

Além desses fatores, as mudanças hormonais típicas da gravidez podem servir como amplificadores desse estresse gestacional (GLAZIER et al, 2004) em razão do aumento do cortisol materno - o hormônio do estresse - que passa a ser produzido também pela placenta a partir do segundo trimestre da gestação (MONTENEGRO et al, 2010).

A elevação nas taxas desse biomarcador tem sido associada a riscos significativos de aborto espontâneo (BAYRAMPOUR et al., 2015), de nascimentos prematuros e consequente baixo peso do bebê (MONK et al., 2000; GLAZIER et al., 2004), além de alterações no desenvolvimento neurocomportamental e padrões fisiológicos relacionados à regulação emocional da criança (KERRY-ANN, MCMAHON \& AUSTIN, 2007; ELLMAN et al., 2008; NATH et al., 2017).

Contextualizando com o momento atual, quando o mundo enfrenta o surto pandêmico do vírus Covid-19, deve-se considerar que os fatores estressores presentes durante o período gestacional estão não apenas sendo amplificados, mas combinados a mais alguns outros, como o medo da gestante de ser infectada pelo vírus, o isolamento necessário da vida social e a incerteza sobre sua duração, a quantidade de informações inadequadas que são passadas diariamente acerca do cenário mundial, assim como a antecipação de uma grave crise econômica em todo o mundo (BROOKS et al., 2020).

Estima-se que aproximadamente $6 \%$ da população de gestantes é acometida por um alto nível de estresse específico da gravidez, o que pode desencadear uma série de problemas (ROMERO-GONZALES et al,2020) como os que foram acima descritos. 
Posto isso, e a partir da consolidação de políticas nacionais para o uso das Práticas Integrativas e Complementares em Saúde (PICS) no Sistema Único de Saúde (SUS) (MINISTÉRIO DA SAÚDE, 2006), foi delineada esta revisão integrativa que tem como objetivo identificar e sistematizar o conhecimento produzido pela literatura científica sobre os efeitos do yoga sobre o estresse em mulheres grávidas. Acredita-se poder ser esta uma estratégia possível de lidar com os mecanismos de estresse e evitar os seus riscos.

Com a ampliação da utilização das PICS (MINISTÉRIO DA SAÚDE, 2017) também conhecidas como Alternative Medicine (WHO, 2002) nos sistemas de saúde globais, outras práticas passaram a ser validadas para os cuidados à saúde, integradas às práticas da medicina tradicional (TESSER, 2009). Uma delas foi o yoga, tradição milenar constituída por um conjunto de exercícios físicos ou ásanas, práticas respiratórias (pranayamas), e de meditação, que promovem estado de relaxamento (FILLA apud RODRIGUES et al., 2006).

O yoga pode ser descrito como uma prática psicofísica que se utiliza de técnicas para controle de funções corporais e mentais (BRASIL, 2018).

Os efeitos psicofísicos desta prática, que instrui técnicas de autocuidado físico e mental (GHAROTE, 2007), têm sido associados à sua influência nos mecanismos do sistema nervoso autônomo, do sistema nervoso central e com ação direta sobre músculos, articulações e vísceras (GHAROTE, 2007), a partir da execução de diferentes posturas físicas, exercícios respiratórios e práticas meditativas (SILVA, 2009). Uma revisão de literatura em 81 pesquisas publicadas no PubMed (ROSS \& THOMAS, 2010) comprovou os benefícios da prática do yoga na ativação do eixo hipotálamo-hipófise-adrenal (HPA) e do Sistema Nervoso Simpático (SNS), repercutindo na saúde mental.

Nessa vertente, uma linha de investigação tem avançado com pesquisas relacionadas aos efeitos do yoga em gestantes no pré-natal. Estudos clínicos controlados indicam que a realização dessa prática no período gravídico reduziu de forma significativa as taxas do cortisol materno - o biomarcador do estresse (FIELD et al, 2013b; SATYAPRIYA et al 2013; ARAUJO 2018; SHAIKH et al., 2013).

Outras evidências clínicas de redução em escores de estresse foram associados, igualmente, a esse tipo de intervenção (SATYAPRIYA et al, 2008), bem como a redução de sintomas e transtornos de ansiedade e depressão (BERSHADSKY et al, 2014; SATYAPRIYA et al., 2013).

Analisando-se estressores psicossociais em gestantes submetidas às intervenções com yoga, foi evidenciada uma associação entre a sua participação em grupos de yoga e um aumento na qualidade da sua saúde psicológica e nas relações interpessoais (SATYAPRIYA et al 2013; QINXIAN et al 2015; FIELD et al., 2012; RAKSHANY et al., 2010).

Na perspectiva da saúde física, a melhora no desconforto e nas dores lombares, considerados estressores físicos, a redução da dor do parto (CHUNTARAPAT et al., 2008) e da sua duração (QINXIAN et al, 2015), também foram resultados correlacionados à prática de yoga na gestação.

Associadas ao estresse, por sofrerem sua influência, as respostas imunológicas de mulheres grávidas obtiveram resultados positivos, que foram correlacionados também às intervenções. Algumas funções vitais do feto também o foram, como a oximetria de pulso, tocometria uterina e frequência cardíaca (MOOVENTHAN, 2019). Foram encontrados resultados positivos também na associação de intervenções com essa prática e a redução de ocorrências de pré-eclâmpsia (FIELD et al., 2013b).

Após as considerações teóricas feitas aqui, uma contextualização torna-se necessária para este estudo. Ele reflete os propósitos do Consórcio Acadêmico Brasileiro de Saúde Integrativa, constituído recentemente pelo Ministério da Saúde, o qual estimula e fomenta pesquisas de diferentes desenhos sobre as PICS (BIREME, 2020). A iniciativa desse consórcio está vinculada ao Departamento de Atenção Básica do referido Ministério e propõe como metas, garantir a gestão do conhecimento produzido pelas citadas pesquisas e a sua integração ao Sistema Único de Saúde (SUS) (OMS, 2013)

Com isso, esta revisão tem dupla relevância. Por um lado atende à demanda por investigações que agreguem conhecimentos ao campo das PICS, e por outro pode servir de referência para profissionais do SUS que desejem implantar em serviços do pré-natal, o trabalho com yoga - um recurso que foi considerado, eficaz para o controle do estresse e promoção da saúde mental de mulheres grávidas, por esta revisão. Muitas usuárias do SUS poderiam beneficiar-se de tais iniciativas. 


\section{Método}

Para este estudo, que se constitui em um subproduto de pesquisa com gestantes adolescentes (ARAÚJO, 2018), foi utilizado o método da revisão integrativa de literatura (WHITTEMORE \& KNAFL, 2005; SOUZA, SILVA \& CARVALHO 2010), iniciada a partir de uma definição clara do seu propósito e, em seguida, com a busca dos artigos científicos utilizando palavras-chave consideradas descritores no DeCS (Descritores em Ciências de Saúde) - yoga; gravidez; pregnancy e estresse.

Essa busca teve início em 2017 (julho-agosto) e finalização em 2020 (agosto). Os critérios de inclusão foram: ser pesquisas originais, publicadas a partir de 2012, cinco anos antes do início desse estudo; apresentar resultados de intervenção com yoga junto a mulheres grávidas e os efeitos dessa prática sobre os sintomas ou marcadores do estresse; integrar na discussão textual os temas gestação-estresse-yoga; ter o texto completo disponível em inglês ou português e estar indexado à base de dados do Sistema Online de Busca e Análise de Literatura Médica e áreas afins da saúde (MEDLINE).

Tendo como norteador, o objetivo de identificar as evidências científicas dos efeitos do yoga sobre o estresse em mulheres grávidas, os dados extraídos por este estudo dos artigos originais pesquisados, foram organizados em uma ficha de coleta que incluía: desenho do estudo, ano, autores e país de publicação, tamanho das amostras (grupos intervenção e controle), faixa etária das participantes, critérios de inclusão nas amostras, atividade do grupo controle, duração das intervenções e os resultados ou efeitos do yoga sobre as gestantes participantes das pesquisas empíricas.

Uma primeira leitura dos artigos levou à identificação de diferentes categorias de respostas às intervenções com yoga nas gestantes dos diversos experimentos. A maior parte dessas respostas estava relacionada aos efeitos da prática sobre sintomas do estresse - objeto deste estudo - que para fins de sistematização teórica, foram aqui designadas como efeitos primários (Tabela 1).

Contudo, outros efeitos que não estavam relacionados diretamente com a questão investigada, foram levantados e analisados como dados secundários os quais, na Tabela 1 estão descritos como "efeitos secundários".
Os impactos da prática de yoga sobre o estresse foram aqui interpretados de acordo com os resultados de cada pesquisa revisada tendo em vista os sintomas apresentados nas gestantes participantes e suas respectivas medidas de avaliação.

Serviram como base para análise dos efeitos daquela prática sobre o estresse nas gestantes participantes, os resultados quantitativos de escores de sintomas nelas mensurados por testes validados e/ou seus relatos bem como questionários aplicados nas pesquisas qualitativas. Também foram analisadas as medidas estatísticas de avaliação dos sintomas entre os grupos intervenção e controle - randomizados ou não randomizados - e nos grupos individuais avaliados em T1 -T2. Nestes casos, os efeitos foram aqui mensurados de acordo com as medidas comparativas inter e intragrupos, tendo como base o nível de significância de $p \leq 0,005$.

\section{Resultados}

A busca levou a um universo de 124 artigos dos quais, após uma leitura exploratória dos resumos, 108 foram descartados por não se adequarem ao tema proposto. Nesses últimos excluídos, o yoga esteve associado a: tabagismo na gestação (1); aspectos biológicos e fisiológicos da gravidez (40); farmacologia para o estresse na gestação (2); modalidades e terapias alternativas na gestação (7); participação do homem durante o processo da gravidez (4); utilização e efeitos gerais do yoga no período gravídico sem o foco no estresse (37) ou uma discussão sobre o estresse sem estar este associado ao yoga ou à gravidez (19). Dos 16 artigos que mostraram adequação ao tema, 3 foram descartados por serem revisões sistemáticas de literatura, de forma que não adequavam ao critério "pesquisa original".

Ao final, 13 artigos que relacionavam a prática do yoga com a gestação e com os sintomas do estresse, foram selecionados para esta revisão. Observa-se uma maior concentração de publicações no ano de 2015 (4 - 30,77\%).

Dos 13 estudos selecionados (Tabela 1), 7 (53,84\%) foram Ensaios Clínicos Randomizados Controlados (ECRC); 2 (15,38\%) Ensaios Clínicos Quase-Experimentais; 2(15,38\%) Ensaios Clínicos Pré e Pós-teste e 2 (15,38\%) Estudos Qualitativos com grupos focais. 


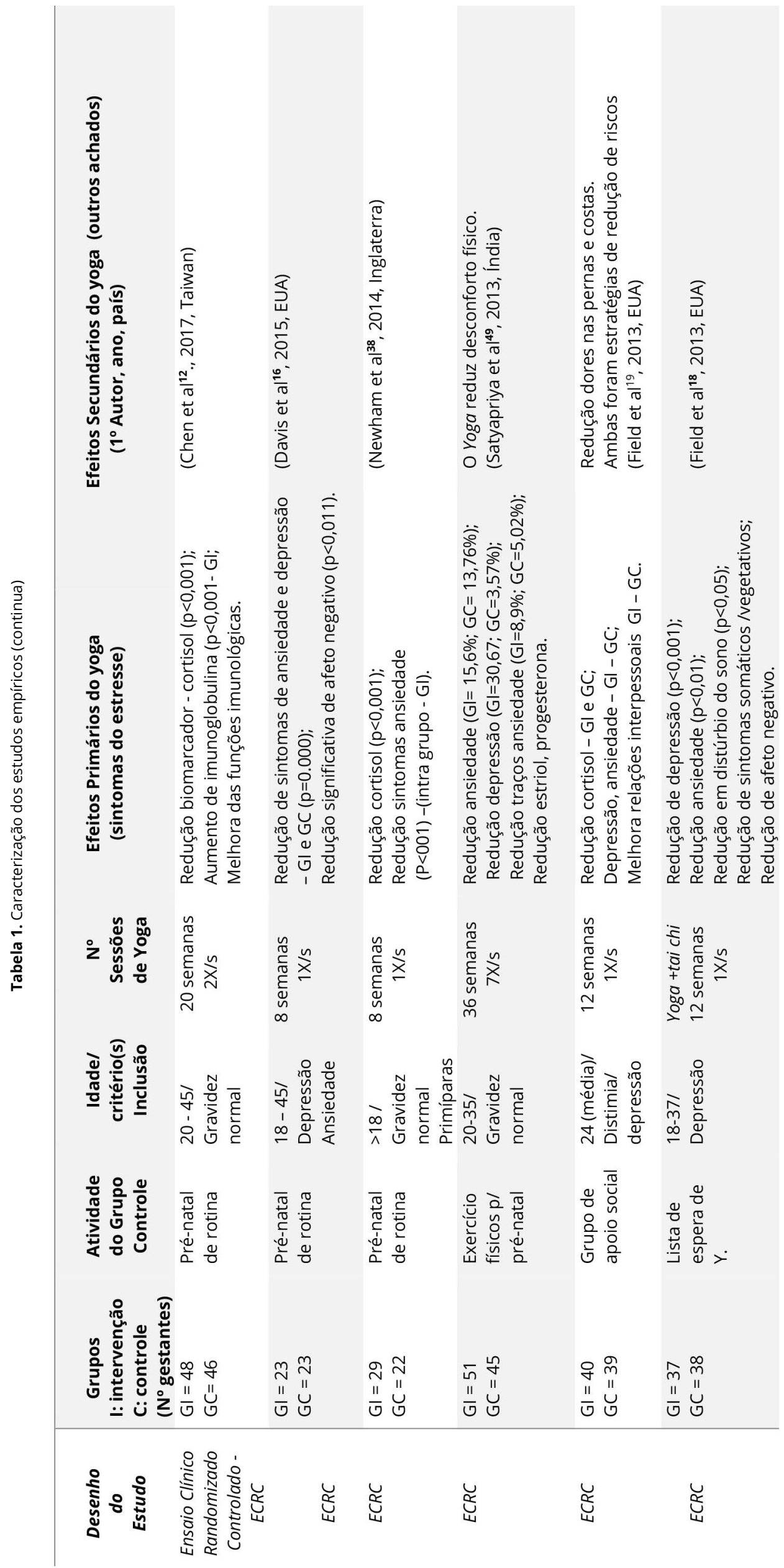



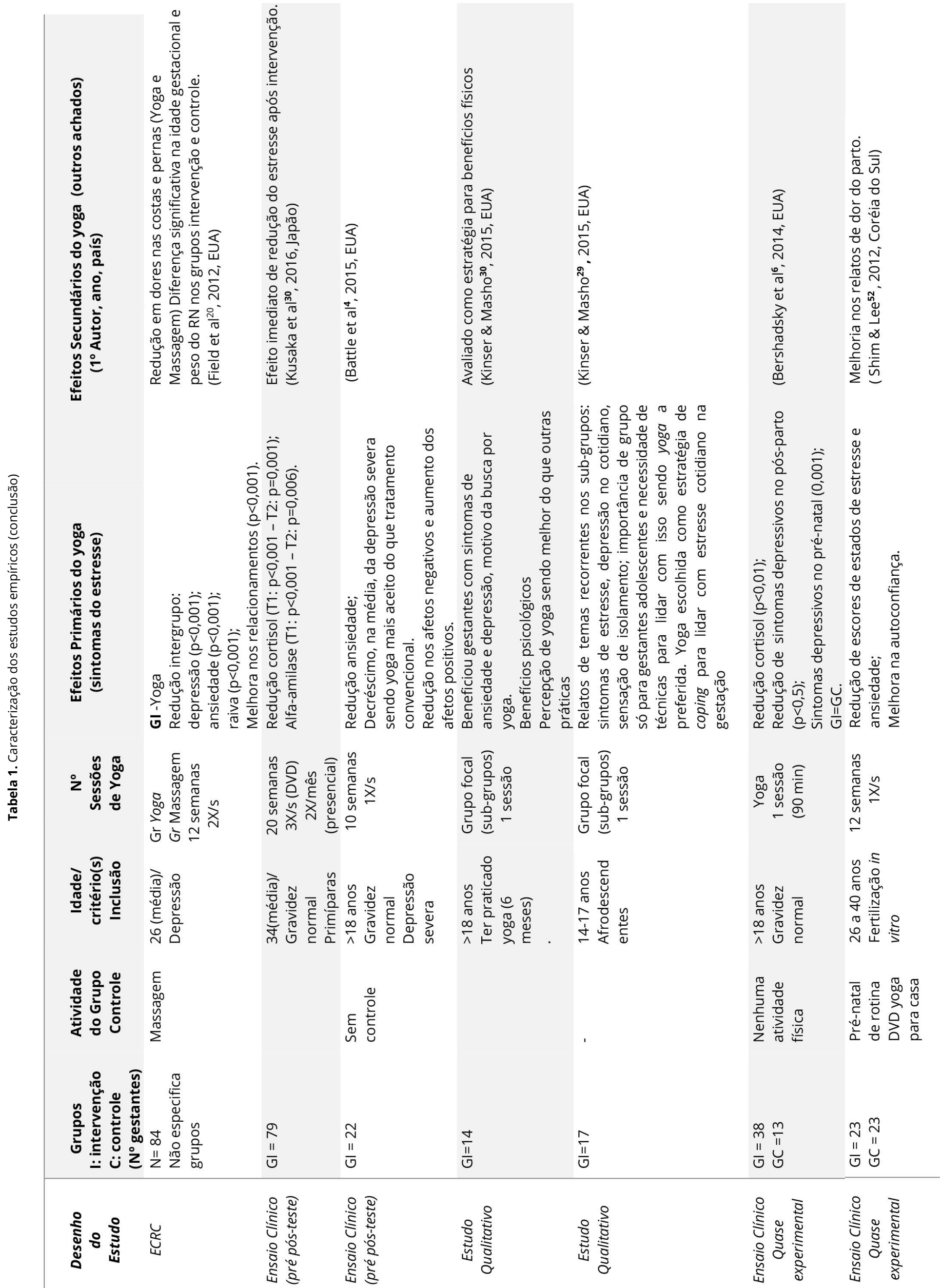
$\mathrm{Na}$ Tabela 1, estão descritos os diferentes países de publicação dos artigos originais - Inglaterra (1), Taiwan (1), Japão (1), Índia (1), Coréia do Sul (1) e Estados Unidos (8), havendo uma concentração neste último. Evidencia-se assim, uma diversidade do local de origem das publicações com o alcance da prática do yoga, com gestantes de diferentes culturas.

Na perspectiva das participantes grávidas somou-se um total de 754 mulheres entre os grupos controle e grupos intervenção havendo, nestes últimos, a participação de $474(62,8 \%)$ gestantes que frequentaram efetivamente, as sessões de yoga. O universo total tinha idade gestacional entre o $2^{\circ}$ e $3^{\circ}$ trimestres de gravidez, e estava na faixa etária entre 14 e 45 anos com predominância entre 20 e 35 anos.

Na perspectiva das intervenções, as sessões de yoga tiveram duração de até 12 semanas e uma variação entre os experimentos, em número de sessões durante a semana. Houve, no entanto, duas exceções a esse padrão. Trata-se de duas pesquisas qualitativas, uma que avaliou os efeitos do yoga em mães que já haviam tido filhos, e que o praticaram na gravidez por conta própria (KINSER \& MASHO, 2015b), e outra, que investigou, por meio de um grupo focal, a autopercepção do estresse por gestantes adolescente, realizando um levantamento sobre preferências de estratégia de coping do estresse, tendo sido o yoga a opção do grupo (KINSER \& MASHO, 2015a).

\section{Efeitos Primários (sintomas do estresse nas gestantes)}

Da identificação e análise dos efeitos primários do yoga nas gestantes já descritos (Tabela 1), emergiram dois grupamentos de sintomas de estresse cujas respostas foram avaliadas após as intervenções: um grupo refletindo a dimensão da saúde mental das participantes, reunindo respostas sobre quadros de humor, aspectos emocionais e relacionais e estresse psicológico global, e outro grupo, expressando a dimensão fisiológica do estresse, agrupando marcadores de respostas do Sistema Nervoso Autônomo e comportamento neurovegetativo no organismo das grávidas.

Concernente à primeira dimensão citada - a da saúde mental - dois quadros de humor são associados, na literatura, a parâmetros de estresse - a ansiedade e a depressão (PAMPKA et al, 2018; NEWHAM et al, 2014). Dados apontaram que sintomas de depressão se constituíram em variável de sete das treze pesquisas desta revisão tendo sito mensurados em 453 (60\%) das participantes entre os grupos controle e intervenção (DAVIS et al., 2015; BATTLE et al., 2015; BERSHADSKY et al., 2014; FIELD et al., 2013a; FIELD et al., 2013b; SATYAPRIYA et al., 2013; FIELD et al., 2012).

Abrangência semelhante tiveram os sintomas de ansiedade, cujos resultados após as intervenções foram avaliados em 499 (66,18\%) gestantes dos grupos intervenção e controle, de oito dos treze estudos (DAVIS et al., 2015; NEWHAM et al., 2014; FIELD et al., 2013a; FIELD et al., 2013b; SATYAPRIYA et al., 2013; FIELD et al., 2012; SHIM \& LEE, 2012; BATTLE et al., 2015).

As pesquisas que incluíram as variáveis de ansiedade e depressão em suas análises, foram ensaios clínicos com grupo controle, com exceção de apenas uma delas (BATTLE et al., 2015) cujas variáveis foram medidas em um grupo individual - antes e após a intervenção.

O diagnóstico prévio desses sintomas nas gestantes participantes dos estudos não foi uma condição definida pela maioria dos protocolos das pesquisas. Daqueles descritos (Tabela 1), cinco definiram a depressão como critério de inclusão (BATTLE et al4, 2015; FIELD et al20, 2012; FIELD et al18, 2013; FIELD et al19, 2013; DAVIS et al16, 2015, EUA) e apenas um definiu o diagnóstico prévio de sintomas ou transtorno de ansiedade para as amostras da investigação (DAVIS et al16, 2015).

As medidas de ambas as variáveis - ansiedade e depressão - apresentaram redução em todos os grupos de gestantes que praticaram o yoga, incluindo a média dos escores de depressão severa (BATTLE et al., 2015) e transtorno de ansiedade (DAVIS et al, 2015) nas gestantes com diagnóstico.

Comparando-se os resultados dos sintomas de alterações em quadros de humor de gestantes que praticaram yoga, com as que não praticaram, dos grupos controle, três destes tiveram medidas igualmente positivas, não havendo diferença com significância estatística entre eles e os grupos de intervenção (DAVIS et I, 2015; FIELD et ala, 2013; NEWHAM et al, 2015). Nestes casos as gestantes do grupo controle contaram com intervenções de apoio social (FIELD et al., 2013b) e orientações, nas consultas de rotina do pré-natal, para a busca de assistência especializada, uma vez que eram gestantes diagnosticadas com sintomas de ansiedade e depressão ( DAVIS et al., 2015; NEWHAM et al, 2015). 
Outros sintomas do estresse nesta dimensão da saúde mental, encontrados entre as participantes das pesquisas, referem-se a aspectos emocionais e relacionais que favoreciam o estresse psicológico, levando-as a disposições negativas do humor.

Alterações positivas foram registradas nessa perspectiva depois das intervenções com yoga tais como: redução de raiva e hostilidade (KUSAKA et al., 2016; FIELD et al., 2013b), de fadiga (KUSAKA et al., 2016), de isolamento (KINSER \& MASHO, 2015b), uma melhora nos índices de afetividade - redução do afeto negativo (FIELD et al., 2013a; DAVIS et al., 2015; BERSHADSKY et al., 2014), aumento do estado de contentamento (BERSHADSKY et al., 2014), e maior confiança da gestante acerca do parto (SHIM \& LEE, 2012).

Foi observado, através de uma análise do discurso das gestantes que participaram de grupos focais (KINSER \& MASHO, 2015b; KINSER \& MASHO, 2015a), o quão presentes são os sintomas de estresse e depressão no seu cotidiano, e que foram apontados, por elas, como fatores que motivaram a escolher a prática do yoga como estratégia em face disso. $\mathrm{Na}$ gravidez, os fatores estressores tendiam a se amplificar, de forma que o estresse acabara por motivar o seu interesse por essa prática no pré-natal (KINSER \& MASHO, 2015a).

O estresse global e não apenas seus sintomas individualmente, foi avaliado por alguns dos estudos (CHEN et al., 2017; NEWHAM et al., 2014; SATYAPRIYA et al., 2012; SHIM \& LEE, 2012). Há evidências de que o yoga teve impacto no nível de estresse, nessa fase, revelando uma redução nos sintomas das gestantes dos grupos de intervenção com yoga em comparação às dos grupos-controle. Enquanto alguns dos estudos apontam uma redução imediata do estresse depois da prática (CHEN et al., 2017; KUSAKA et al., 2016), outros relatam a sua manutenção ao longo das intervenções (KINSER \& MASHO, 2015a; SHIM \& LEE, 2012). Os seus sintomas foram reduzidos significativamente (KINSER \& MASHO, 2015b)

Na dimensão fisiológica, o foco de algumas pesquisas esteve na aferição dos níveis de cortisol das gestantes, sendo este hormônio o biomarcador mais utilizados como um dos sintomas, no corpo, do estresse, segundo os artigos revisados (CHEN et al., 2017; NEWHAM et al., 2014; FIELD et al., 2013b; KUSAKA et al., 2016; BERSHADSKY et al., 2014), apresentando uma redução de suas medidas nos grupos de yoga, se comparados com os grupos controle, de variação de $p<0,01$ a $p<0,001$.

Dois daqueles estudos, além do cortisol, avaliaram outros biomarcadores: a alfa-amilase salivar, com redução avaliada em um experimento pré e pós-teste de $p<0,001$ (T1) e $p=0,006$ (T2) (KUSAKA et al., 2016), a progesterona e o estriol, medidos em um estudo controlado e randomizado (FIELD et al., 2013b), indicando uma redução depois da última sessão de yoga, embora sem significância, no grupo de intervenção.

Os autores desse estudo (FIELD et al., 2013b) revelaram que as relações entre cortisol, progesterona e estriol ainda estavam claras, uma vez que dois desses biomarcadores (progesterona e estriol) apresentaram um aumento dos seus níveis entre o primeiro e último dia de intervenção, em ambos os grupos-controle e intervenção, e uma redução dos seus níveis apenas depois da prática final.

Os sintomas de perturbações do sono, também agrupado nesta dimensão da fisiologia do estresse, sendo uma função regulada pelo sistema neurovegetativo ou sistema nervoso autônomo, foi avaliado por Field et al. (2012) Esse estudo, utilizou como intervenção tanto a prática de yoga associada ao tai-chi - ambas já consolidadas como PICS - encontrando nesse grupo uma redução nos níveis de perturbação do sono, em comparação com o controle $(p<0,05)$.

Esta prática do yoga pré-natal foi identificada como um método adequado para manejo do estresse não sendo encontrados efeitos adversos nos estudos aqui revistos.

\section{Efeitos Secundários (dor e desconforto na gestação e parto e marcadores de saúde do recém-nascido)}

Conforme citado anteriormente, outros efeitos que não estavam relacionados diretamente a parâmetros do estresse, também foram identificados nos estudos com yoga sendo considerados nesta investigação teórica como dados secundários e identificados como efeitos secundários, em sua sistematização (Tabela 1).

Os efeitos mais recorrentes estavam relacionados a dores e desconforto físicos na gestação e, em apenas duas pesquisas, a aferição de indicadores vitais de saúde do recém-nascido. 
Na análise dos artigos estudados observou-se que a avaliação de dor física indicou que a prática do yoga pré-natal provoca efeitos fisiológicos benéficos ao corpo. Foram citadas a redução da dor em seus diversos domínios, como a dor do parto (SHIM \& LEE, 2012) e as dores físicas - costas e pernas - durante a gestação (FIELD et al., 2013b; FIELD et al., 2012), bem como uma melhora nos parâmetros do parto (CURTIS et al., 2012).

Na linha de mais um estudo utilizando outra modalidade de PICS além do yoga, um dos autores (FIELD et al.,2012) apresentou o efeito positivo da massagem associada ao yoga no que tange às dores físicas relativas à gravidez - principalmente na lombar e nas pernas - e ressalta que o yoga, devido às suas posturas e aos seus movimentos, pode ser considerada como uma prática de automassagem.

Também resultados melhores foram encontrados nessa investigação, com relação à idade gestacional e ao peso do recém-nascido.

\section{Discussão}

A descrição e o alinhamento dos resultados dos efeitos do yoga avaliados nas gestantes, conforme expostos na Tabela 1, demonstra uma diversidade de sintomas de estresse, que afetaram tanto a saúde mental dessas mulheres como aspectos orgânicos considerando a sua fisiologia. Esta diversidade de sintomas é uma característica dos atributos do estresse, decifrada por Selye (1950) como uma síndrome, cujos parâmetros são mantidos pela ciência até os dias atuais. Seus estudos explicitaram que o ser humano pode ser ativado por estressores de vetores físicos, psíquicos ou ambientais, provocando o estado de estresse.

Em caso de ser o estresse agudo ou intermitente, ele provocar alterações no funcionamento do Sistema Nervoso Autônomo, que regula o eixo hipotálamo-pituitária-adrenal (HPA) levando isto, a uma produção exacerbada do hormônio cortisol, que passou a ser reconhecido como o hormônio do estresse, dado a sua abundância nesses casos (SELYE, 1950).

Durante a gestação, o eixo HPA apresenta um aumento de sua ativação gerando uma elevada concentração de cortisol (JURUENA et al. 2004). Observou-se, nesta revisão, que os estudos que avaliaram esse hormônio associaram a prática do yoga ao funcionamento positivo desse eixo, uma vez que foi identificada uma queda do nível da concentração do cortisol nas gestantes dos grupos de intervenção como já citado(CHEN et al. 2017; KUSAKA et al. 2016; BERSHADSKY et al. 2014; NEWHAM et al., 2014; FIELD et al 2013b).

Isso tem relevância na ciência, pois estudos apontam que o estresse está associado a níveis altos de cortisol em gestante podendo isto levar a resultados negativos pré e perinatais, tais como: aborto espontâneo (BAYRAMPOUR et al., 2015), nascimento prematuro e consequente peso abaixo da média (NEWTON et al., 1979; MONK et al., 2000; GLAZIER et al., 2004).

Além do cortisol, a prática do yoga pré-natal também foi associada à redução na concentração salivar da alfa-amilase salivar (KUSAKA et al., 2016), a ptialina, usada como um biomarcador alternativo à noradrenalina e que naturalmente tem a sua reatividade ao estresse atenuada durante o terceiro trimestre, entretanto, com a prática do yoga, a sua concentração reduziu consideravelmente no fim de cada prática (KUSAKA et al., 2016).

Ainda na dimensão fisiológica do estresse, a literatura aponta um aumento do nível de imunoglobulina A - um anticorpo - imediatamente depois da prática de yoga (CHEN et al., 2017), o que revela o impacto dessa prática nas funções imunológicas das gestantes, gerando uma melhoria no seu sistema imunológico. Isso é especialmente importante na atualidade com o fenômeno pandêmico do vírus Covid-19 (WHO, 2020), quando a gestante foi recentemente colocada entre os grupos de risco (OPAS, 2020) e os níveis de estresse vêm aumentando exponencialmente na população como um todo, devido aos seus impactos (BROOKS et al., 2020).

A repercussão positiva da prática de yoga em biomarcadores do estresse no organismo da gestante reforça a associação entre essa prática com um meIhor funcionamento do HPA e, consequentemente, do funcionamento do sistema nervoso autônomo (CURTIS et al. 2012). É possível que aquela prática também esteja associada à uma menor incidência de distúrbios pré-natais, pactuando com outros autores (CHEN et al. 2017; CURTIS et al. 2012; FIELD et al. 2013a; QINXIAN et al., 2015).

Estudos teóricos que resgatam a origem do yoga (GHAROTE, 2007; SILVA, 2009) descrevem os seus 
efeitos psicofísicos, resultantes da execução de posturas físicas específicas, exercícios respiratórios controlados e práticas meditativas, e afirmam que estes são capazes de interferir em mecanismos autônomos do sistema neurovegetativo (GHAROTE, 2007) que atuam no eixo Hipotálamo-pituitária-adrenal (HPA). É nesta associação entre o sistema de práticas do yoga e o funcionamento do sistema nervoso autônomo que acredita-se residir os efeitos positivos identificados nas gestantes após as intervenções.

No âmbito da saúde mental os resultados revelaram que os sintomas de estresse identificados como mais recorrentes nas gestantes dos estudos empíricos - o de ansiedade e depressão - tornaram-se significativamente menores depois das intervenções com yoga. Nesse sentido, foi encontrado, na literatura, uma prevalência alta variando de 12,6 a $19,6 \%$ na comorbidade desses transtornos ao longo da gravidez (LEE et al. 2007), o que provavelmente explica terem sido eles variáveis de análise em onze das treze pesquisas desta revisão.

Elas revelaram algumas vantagens da prática do yoga em gestantes com quadros depressivos ou sintomas de depressão. Os dados indicaram, além de uma redução em todos os grupos com as intervenções, também correlacionaram efeitos positivos do yoga, e mais especificamente, de suas modalidades - o Hatha Yoga - com a redução de sintomas de depressão pós-parto (BERSHADSKY et al., 2014), demonstrando ser eficaz para as participantes clinicamente já diagnosticadas.

Observou-se, na literatura, que o estresse e a depressão apresentam uma grande incidência na vida diária de mulheres grávidas, de forma que eles foram associados por alguns autores como fatores que incentivam muitas mulheres a buscar e a fazer adesão ao yoga pré-natal (KINSER \& MASHO, 2015a; KINSER \& MASHO, 2015b).

Há uma demanda por esse tipo de prática que oferte espaço para o desenvolvimento de atividades integrativas em grupo, o controle do estresse, depressão e melhoria das relações interpessoais (KINSER \& MASHO, 2015a; KINSER \& MASHO, 2015b; BERSHADSKY et al., 2014; NEWHAM et al., 2014).

Os resultados indicaram que mulheres diagnosticadas com depressão creditaram à prática no grupo de yoga a melhora dos seus quadros depressivos, indicando, de igual forma, seu nível alto de satisfação com essa prática (KINSER \& MASHO, 2015a). Foi associada também a altos níveis de credibilidade e satisfação como uma intervenção para ansiedade e estresse durante a gravidez, sendo entendido como benefício, tanto no âmbito psicológico como no físico (KINSER \& MASHO, 2015a; QINXIAN et al., 2015).

Ainda no âmbito da saúde mental, através deste estudo, foi possível constatar que gestante de alguns grupos de intervenção relataram uma melhora em suas relações interpessoais (QINXIAN et al., 2015; FIELD et al., 2013b; CURTIS et al., 2012; FIELD et al., 2012), o que pode ser creditado à redução de aspectos negativos do humor, uma melhora em sua qualidade de vida (CURTIS et al., 2012) e maior autoconfiança (SHIM \& LEE, 2012). Um dos estudos, entretanto, apresentou melhora nas relações interpessoais também no grupo-controle, que consistia em um grupo de apoio social (FIELD et al., 2013b), revelando a importância desse fator. Isso sinaliza a importância do grupamento social com outras gestantes, o que promove um sentimento de pertencimento, fortalecendo, por conseguinte, sua autoestima.

Além da discussão sobre os efeitos primários relacionados aos sintomas do estresse que foram encontrados, torna-se relevante destacar aqui a importância dos efeitos secundários provocados pela prática de yoga sobre dores e o desconforto físico nas mulheres grávidas.

Apesar da etiologia de tais sintomas não residirem no estresse e sim em uma condição anátomo-fisológica, elas podem se constituir em fatores estressores e desencadear o mecanismo do estresse. Este mecanismo surge da necessidade do indivíduo de lidar com fatores externos ou internos ao organismo - que podem ser as dores físicas - as quais, enquanto estressores são capazes de criar tensões e desdobramentos identificados como efeitos primários (SADIR, BIGNOTTO \& LIPP, 2010; SELYE, 1950).

Segundo Sperandio et al. (2004), 50\% a 75\% das gestantes experimentam dores nas costas durante a gestação que podem variar conforme tipos, áreas e intensidade, podendo alcançar tamanha intensidade que se torne incapacitante para as mulheres, em determinadas situações. Na literatura, as dores mais comumente citadas foram as de coluna, lombar, pélvis e pernas, assim como dor no parto (FIELD et al., 2013; ; FIELD et al., 2013b; FIELD et al. 2012; SHIM \& LEE, 2012).

A gestação é um momento na vida da mulher em que mente e corpo, devem se adaptar às novas 
demandas. Nos artigos podem-se encontrar relatos de gestantes sobre como ele perpassa o seu dia a dia gerando sintomas em diferentes dimensões - tanto física como psíquica (KINSER \& MASHO, 2015a; KINSER \& MASHO, 2015b; KUSAKA et al., 2016).

Sendo o yoga um sistema de práticas corpo/mente que favorece a uma homeostase mediante o controle de funções orgânicas e mentais, abrangendo os sistemas, músculos e vísceras (SILVA, 2009), acreditase ter sido esta abrangência do seu alcance no ser humano o que o tornou uma estratégia favorável ao controle dos sintomas de estresse pelas gestantes, reduzindo os seus riscos.

\section{Conclusão}

O yoga é uma prática que integra corpo e mente, de forma que atinge as questões fisiológicas - de funcionamento do corpo - e emocionais do indivíduo, acompanhando a atual tendência de cuidados com a saúde, conforme discutido pela Organização Mundial da Saúde (OMS), de que o cuidado deve ser integral e o indivíduo deve ser visto e entendido através de uma perspectiva holística.

Este artigo demonstra a eficácia e a efetividade da prática do yoga no pré-natal, não tendo sido encontrados efeitos adversos. Os benefícios levantados, sobretudo aqueles referentes aos quadros clínicos de depressão e ansiedade, colocam-na como uma estratégia que pode ser prescrita, tanto de forma preventiva como complementar no tratamento dos sintomas e transtornos de humor na gestação.

Como mencionado anteriormente, atualmente, o mundo vive um momento atípico. Com o surto pandêmico do Covid-19, o consequente distanciamento social e o isolamento social - enquanto medidas de segurança, a incidência de transtornos como ansiedade e depressão, bem como os seus sintomas e traços vêm aumentando exponencialmente.

Isso pode estar potencializado na população de gestantes, uma vez que elas necessitam utilizar os serviços de assistência à saúde - considerados de alto risco para a contaminação - para a realização do seu pré-natal e futuro parto. Tal fato pode fazer crescer a incerteza acerca do futuro, que antes, concentrado nas expectativas da gestação, agora abrange toda uma instabilidade social, econômica e ambiental em nível mundial. Ressalte-se aqui que entre esta população das mulheres gestantes, existe uma parcela de adolescentes cuja condição da gravidez precoce algumas vezes de risco, favorece a condição de estresse persistente.

Em face disto, sugere-se que novas pesquisas sejam desenvolvidas visando avaliar a intervenção do yoga em pacientes gestantes contaminadas por Covid-19, fazendo-se uso de tecnologias virtuais, bem como por gestantes em geral, na mesma modalidade virtual, enquanto instrumento para controle dos impactos psicológicos do confinamento e isolamento social

Recomenda-se também o desenvolvimento de ensaios clínicos randomizados com gestantes portadoras de transtornos depressivos e de ansiedade avaliando o controle dos sintomas, tanto com a prática do yoga como por substâncias psicoativas necessárias de prescrição. Além disso, sugere-se que estudos sejam realizados para avaliar a viabilização, em serviços públicos de saúde, de grupos de yoga no pré-natal e que este trabalho se constitua em mais uma contribuição para o corpo de conhecimento que o Consórcio Acadêmico Brasileiro de Saúde Integrativa tem gerado para apoiar as práticas de cuidados na Atenção Básica, podendo beneficiar, com isto, a usuárias do SUS.

\section{Limitações do estudo}

O estudo foi feito em apenas uma base de dados. Esta é uma pesquisa preliminar que analisa os efeitos do yoga em mulheres gestantes a partir de outros estudos publicados no período de 2012-2019. Para resolver essas limitações, sugere-se que seja feita uma revisão sistemática do tema, apresentando uma ampliação dos resultados, incluindo anos posteriores.

\section{Contribuições das autoras}

Araújo MAN concebeu a pesquisa matriz que direcionou a elaboração desta revisão que se constituiu em um plano de trabalho de uma discente de Iniciação Científica. Definiu o formato da busca de artigos para a revisão, orientando a coleta e criação do instrumento de tabulação para tratamento dos dados. Orientou a interpretação dos mesmos bem como a redação do artigo. Prado BGL elaborou, como discente de Iniciação Científica, o plano de trabalho inicial para esta revisão, realizou as buscas nas bases de dados coletando-os seguindo os critérios de inclusão estabelecidos no plano, tabulou e organizou todo o material coletado para a interpretação dos resultados, participou ativamente da análise destes últimos e da redação do artigo científico. 


\section{Conflitos de interesses}

Nenhum conflito financeiro, legal ou político envolvendo terceiros (governo, empresas e fundações privadas, etc.) foi declarado para nenhum aspecto do trabalho submetido (incluindo, mas não se limitando a subvenções e financiamentos, participação em conselho consultivo, desenho de estudo, preparação de manuscrito, análise estatística, etc.).

\section{Referências}

Ahn, H. L. (1984). An experimental study of the effects of husband's supportive behavior reinforcement education on stress relief of primigravidas. Journal of Korean Academy of Nursing, 15(1), 5-16. https://www.koreamed.org/ SearchBasic.php?RID=2326547

Arantes, M. A. A. C., \& Vieira, M. J. F.(2002). Estresse: clínica psicanalítica $\left(3^{\mathrm{a}}\right.$. ed). Casa do psicólogo.

Araújo, M. A. N., Aguiar, C. V. N., Powell V. M. B., Silva, S. M. B., Nunes, A. C. R., Goes, A. L. B., ... Pondé, M. P. (2018). Intervenção com yoga em gestantes adolescentes: efeitos sobre estressores e sobre parâmetros do estresse (Tese de Doutorado, Programa de Pós Graduação da Escola Bahiana de Medicina e Saúde Pública). https://repositorio. bahiana.edu.br:8443/jspui/handle/bahiana/3898

Battle, C. L., Uebelacker, L. A., Magee, S. R., Sutton, K. A., \& Miller, I. W. (2015). Potential for prenatal yoga to serve as an intervention to treat depression during pregnancy. Womens Health Issues, 25(2), 134-41. https://doi. org/10.1016/j.whi.2014.12.003

Bayrampour, H., Salmon C., Vinturache, A., \& Tough, S. C. (2015). Effect of depressive and anxiety symptoms during pregnancy on risk of obstetric interventions. Journal of Obstetrics and Gynaecology Research. Journal of Obstetrics and Gynaecology Research, 41(7), 1040-1048.. https://doi. org/10.1111/jog.12683

Bershadsky, S., Trumpfheller L., Kimble, H.B., Pipaloff, D., \& Yim, I.S. (2014). The effect of prenatal Hatha yoga on affect, cortisol and depressive symptoms. Complementary Therapies in Clinical Practice, 20(2),106-13. https://doi. org/10.1016/j.ctcp.2014.01.002

BIREME. (2019). Consórcio de pesquisadores contribui para qualificar as evidências sobre saúde integrativa. Boletim BIREME, 29. http://boletin.bireme.org/pt/2019/03/04/ consorcio-de-pesquisadores-contribui-para-qualificar-asevidencias-sobre-saude-integrativa/

Brasil. Ministério da Saúde (2006). Secretaria de Atenção à Saúde. Departamento de Atenção Básica. Política Nacional de Práticas Integrativas e Complementares (1a ed). Ministério da Saúde. p. 10. https://bvsms.saude.gov.br/bvs/ publicacoes/pnpic.pdf
Brasil. Ministério da Saúde. (2018). Glossário Temático: Práticas Integrativas e Complementares em Saúde. Biblioteca Virtual em Saúde do Ministério da Saúde. Ministério da Saúde. Disponível em: http://portalarquivos2.saude.gov.br/ images/pdf/2018/marco/12/glossario-tematico.pdf

Brooks, S. K, Webster, R. K., Smith, L. E., Woodland, L., Wessely, S., Greenberg, N.,... Rubin, G. J. (2020). The psychological impact of quarantine and how to reduce it: rapid review of the evidence. The Lancet, 395(10227),912-920. https://doi. org/10.1016/S0140-6736(20)30460-8

Chen, P. J., Yang, L., Chou C. C., Li, C. C., Chang, Y. C., \& Liaw, J. J. (2017). Effects of prenatal yoga on women's stress and immune function across pregnancy: A randomized controlled trial. Complementary Therapies in Medicine, 31,109-117. https://doi.org/10.1016/j.ctim.2017.03.003

Choi, Y. S. (1983). An experimental study of the effects of childbirth preparation on labor pain (Unpublished doctoral dissertation), Yonsei University.

Chuntharapat, S., Petpichetchian, W., \& Hatthakit, U. (2008). Yoga duringpregnancy: effects on maternal comfort, labor pain and birthoutcomes. Complementary Therapies in Clinical Practice, 14(2):105-15. https://doi.org/10.1016/j. ctcp.2007.12.007

Curtis, K., Weinrib, A., \& Katz, J. (2012). Systematic review of yoga for pregnant women: current status and future direction. Evidence-Based Complementary and Alternative Medicine. https://doi.org/10.1155/2012/715942

Davis, K., Goodman, S.H., Leiferman, J., Taylor, M., \& Dimidjian, S. (2015). A randomized controlled trial of yoga for pregnant women with symptoms of depression and anxiety. Complementary Therapies in Clinical Practice, 21(3):166-72. https://doi.org/10.1016/j.ctcp.2015.06.005

Ellman, L.M., Schetter, C.D., Hobel, C.J., Chicz-Demet, A., Glynn, L.M. \& Sandman, C.A. (2008). Timing of fetal exposure to stress hormones: effects on newborn physical and neuromuscular maturation. Developmental Psychobiology, 50(3),232-41. https://doi.org/10.1002/dev.20293

Field, T., Diego M., Delgado, J., \& Medina, L. (2013)a. Tai chi/ yoga reduces prenatal depression, anxiety and sleep disturbances. Complementary Therapies in Clinical Practice, 19(1),6-10. https://doi.org/10.1016/j.ctcp.2012.10.001

Field, T., Diego M., Delgado J., \& Medina L. (2013)b. Yoga and social support reduce prenatal depression, anxiety and cortisol. J Bodyw Mov Ther, 17(4),397-403. https://doi.org/10.1016/j. jbmt.2013.03.010

Field, T., Diego M., Hernandez-Reif, M., Medina L., Delgado J., \& Hernandez A. (2012). Yoga and massage therapy reduce prenatal depression and prematurity. Journal of bodywork and movement therapies, 16(2), 204-9. https://doi. org/10.1016/j.jbmt.2011.08.002 
Filla, J. A. M. (2006). Yoga-Nidrã. A ciência do relaxamento. In M. R. Rodrigues, C. Deveza, D. F. Santella, J. A. M. Filla, L. C. Gulmini, M. A. C. Di Benedetto, M. C. Castilho, M. Ferreira, \& S. Shimada (Orgs). Estudos sobre o Yoga. Phorte.

Gharote, M. L. (2007). Técnicas de Yoga (2 $2^{\mathrm{a}}$ ed.) Phorte Editora.

Glazier, R. H., Elgar, F. J., Goel, V., \& Holzapfel, S. (2004). Stress, social support, and emotional distress in a community sample of pregnant women. Journal of psychosomatic obstetrics \& gynecology, 25(3-4), 247-55. https://doi. org/10.1080/01674820400024406

Juruena, M. F., Cleare, A. J., \& Pariante, C. M. (2004). The hypothalamic pituary adrenal axis, glucocorticoid receptor function and relevance to depression. Revista Brasileira de Psiquiatria, 26(3), 189-201. https://doi.org/10.1590/S151644462004000300009

Engliston, K. A., McMahon, C., \& Austin, M. P. (2007).

Stress in pregnancy and infant HPA axis function: Conceptual and methodological issues relating to the use of salivary cortisol as an outcome measure. Psychoneuroendocrinology, 32(1), 1-13. https://doi. org/10.1016/j.psyneuen.2006.10.003

Kim, J., Shin, D. K., \& Shin, G. (1978). A study based on the standardization of the STAl for Korea. The New Medical Journal, 21(11), 69-75. https://bit.ly/32h7c1j

Kinser, P., \& Masho, S. (2015)a. "I Just Start Crying for No Reason": The Experience of Stress and Depression in Pregnant, Urban, African-American Adolescents and Their Perception of Yoga as a Management Strategy. Women's Health Issues, 25(2), 142-8. https://doi.org/10.1016/j.whi.2014.11.007

Kinser, P., \& Masho, S. (2015)b. "Yoga Was My Saving Grace" The Experience of Women Who Practice Prenatal Yoga. Journal of the American Psychiatric Nurses Association, 21(5):319-26. https://doi.org/10.1177/1078390315610554

Kusaka, M., Matsuzaki, M., Shiraishi M., \& Haruna, M. (2016). Immediate stress reduction effects of yoga during pregnancy: One group pre-post test. Women and Birth, Journal of the Australian college of Midwives, 29(5), e82-e88. https://doi.org/10.1016/j.wombi.2016.04.003

Lee, A. M., Lam, S.K., Lau, S. M. S. M., Chong, C. S. Y., Chui, H. W., \& Fong, D. Y. T. (2007). Prevalence, Course, and Risk Factor for Antenatal Anxiety and Depression. American College of Obstetrics \& Gynecology, 110(5), 1102-1112. https://doi. org/10.1097/01.AOG.0000287065.59491.70

Lee, M. K. (2003). Effects of San-Yin-Jiao(SP6) acupressure on labor pain, delivery time in women during labor. Journal of Korean Academy ofNursing, 33(6), 753-761. https://doi. org/10.4040/jkan.2003.33.6.753
Lindsay, J. R., \& Nieman, L. K. (2005). The hypothalamic-pituitaryadrenal axis in pregnancy: challenges in disease detection and treatment. Endocrine reviews, 26(6), 2004-25. https:// doi.org/10.1210/er.2004-0025

Monk, C., Fifer, W.P., Myers, M. M., Sloan, R. P., Trien, L., \& Hurtado, A. (2000). Maternal Stress responses and anxiety during pregnancy: Effects on fetal heart rate. Dev. Psychobiol, 36:67-77. https://doi.org/10.1002/(SICl)10982302(200001)36:1<67::AID-DEV7>3.0.CO;2-C

Montenegro, A. C. P., D' Assunção, V. R., Luna, M. G. B., Raposo, P. V. N., \& Bandeira, F. (2010). Evaluation of levels of cortisol in saliva using electro-chemical luminescence in low-risk and high-risk pregnancies. Revista Brasileira de Saúde Materno Infantil, 10(1), 69-74. https://dx.doi.org/10.1590/ S1519-38292010000100007

Mooventhan, A. (2019). A comprehensive review on scientific evidence-based effects (including adverse effects) of yoga for normal and high-risk pregnancy-related health problems. Journal of Bodywork \& Movement Therapies (23), 721-727. https://doi.org/10.1016/j.jbmt.2019.03.005

Nath, A., Murthy, G.V.S., Babu, G.R., \& Renzo, G. C. D. (2017). Effect of prenatal exposure to maternal cortisol and psychological distress on infant development in Bengaluru, southern India: a prospective cohort study. BMC Psychiatry, 17(1), 255. https://doi.org/10.1186/s12888017-1424-x

Newham, J. J., Wittkowski, A., Hurley, J., Aplin, J. D., \& Westwood M. (2014). Effects of antenatal yoga on maternal anxiety and depression: a randomized controlled trial. Department of Midwifery and Women's Health, 31(8), 631-40. https://doi. org/10.1002/da.22268

Organización Mundial de la Salud (OMS) (2013). Estrategia de la OMS sobre medicina tradicional. Catalogación por la Biblioteca de la OMS. https://apps.who.int/iris/bitstream/ handle/10665/95008/9789243506098_spa.pdf;jsessioni$d=4622184$ A0F2921 E8BED760C27A5183D4?sequence $=1$

Organização Pan-Americana de Saúde (OPAS). (2020). OPAS pede aos países que garantam controle pré-natal a gestantes devido ao risco de COVID-19 grave. OPAS Brasil, Banco de Notícias. https://www.paho.org/bra/index. php?option=com_content\&view=article\&id=6256:op as-pede-aos-paises-que-garantam-controle-pre-natal-agestantes-devido-ao-risco-de-covid-19-grave\&Itemid=820

Pampaka, D., Papatheodorou, S. I., AlSeaidan, M., Wotayan, R. A., Wright, R. J., Buring, J. E., ... Christophi C. A. (2018). Postnatal depressive symptoms in women with and without antenatal depressive symptoms: results from a prospective cohort study. Archives of Women's Mental Health, 22(1), 93-103. https://doi.org/10.1007/s00737-0180880-8 
Portaria n. 849, de 27 de março de 2017. Inclui a Arteterapia, Ayurveda, Biodança, Dança Circular, Meditação, Musicoterapia, Naturopatia, Osteopatia, Quiropraxia, Reflexoterapia, Reiki, Shantala, Terapia Comunitária Integrativa e Yoga à Política Nacional de Práticas Integrativas e Complementares. Diário Oficial da União. https://bvsms.saude.gov.br/bvs/saudelegis/gm/2017/ prt0849_28_03_2017.html

Jiang, Q., Wu, Z., Zhou, L., Dunlop, J., \& Chen, P. (2015). Effects of yoga intervention during pregnancy: a review for current status. Thieme, American Journal of perinatology, 32(6), 50314. https://doi.org/10.1055/s-0034-1396701

Rakhshani, A., Maharana, S., Raghuram, N., Nagendra, H. R., \& Venkatram, P. (2010). Effects of integrated yoga on quality of life and interpersonal relationship of pregnant women. Qual Life Res, 19(10), 1447-55. https://doi.org/10.1007/ s11136-010-9709-2

Rodrigues, O. M. P. R., \& Schiavo, R. A. (2011). Stress na gestação e no puerpério: uma correlação com a depressão pós-parto. Revista Brasileira de Ginecologia e Obstetrícia, 33(9), 252257. https://doi.org/10.1590/S0100-72032011000900006

Rodrigues, M. R. (2006). O que é Yoga? In M. R. Rodrigues, C. Deveza, D. F. Santella, J. A. M. Filla, L. C. Gulmini, M. A. C. Di Benedetto, M. C. Castilho, M. Ferreira, \& S. Shimada (Orgs). Estudos sobre o Yoga. Phorte.

Romero-Gonzales, B., Puertas-Gonzales, J. A., Strivens-Vilchez, H., Gonzalez-Perez, R., \& Peralta-Ramirez, M.I. (2020). Effects of cognitive-behavioural therapy for stress management on stress and hair cortisol levels in pregnant women: A randomized controlled trial. Journal of Psychosomatic Research, 135. https://doi.org/10.1016/j. jpsychores.2020.110162

Ross, A., \& Thomas, S. (2010). The health benefits of yoga and exercise: A review of comparison studies. Journal of Alternative and Complementary Medicine, 16(1), 3-12. https://doi.org/10.1089/acm.2009.0044

Sadir, M. A., Bignotto, M. M., \& Lipp, M. E. N. (2010). Stress e qualidade de vida: influência de algumas variáveis. Paideia, 20(45), 73-81. https://doi.org/10.1590/S0103$863 \times 2010000100010$

Satyapriya, M., Nagarathna, R., Padmalatha, V., \& Nagendra, H.R. (2013). Effect of integrated yoga on anxiety, depression \& well bein in normal pregnancy. Complementary Therapies in Clinical Practice, 19(4), 230-6. https://doi.org/10.1016/j. ctcp.2013.06.003
Satyapriya, M, Nagendra, H. R., Nagarathna, R., \& Padmalatha, V. (2008). Effect of integrated yoga on stress and heart rate variability in pregnant women. International Journal of Gynecology \& Obstetrics, 104(3), 218-22. https://doi. org/10.1016/j.ijgo.2008.11.013

Selye, H. (1950) Stress and the General Adaptation Syndrome. British Medical Journal, 1(4667), 1383-1392. https://doi. org/10.1136/bmj.1.4667.1383

Shaikh, K., Premji S, Khowaja, K., Tough, S., Kazi, A., \& Khowaja, S. (2013). The Relationship between Prenatal Stress, Depression, Cortisol and Preterm Birth: A Review. Open Journal of Depression, 2(3), 24-31 http://dx.doi.org/10.4236/ ojd.2013.23006

Shim C. S., \& Lee Y.S. (2012). Effects of a yoga-focused prenatal program on stress, anxiety, self confidence and labor pain in pregnant women with in vitro fertilization treatment. Journal of Korean Academy of Nursing, 42(3), 369-76. https:// doi.org/10.4040/jkan.2012.42.3.369

Silva, G. D., \& Lage, L. V. (2006). loga e fibromialgia. Revista Brasileira de Reumatologia, 46(1), 37-39. https://doi. org/10.1590/S0482-50042006000100008

Souza, M. T., Silva, M. D., \& Carvalho, R. (2010). Revisão integrativa: o que é e como fazer. Integrative review: what is it? How to do it? Einstein, 8(1), 102-6. https://doi.org/10.1590/s167945082010 rw1134

Sperandio, F. F., Santos, G. M., Pereira, F. (2004). Características e diferenças da dor sacroilíaca e lombar durante a gestação em mulheres primigestas e multigestas. Fisioterapia Brasil 5(4), 267-271. http://dx.doi.org/10.33233/fb.v5i4.3155

Tesser, C. D. (2009). Práticas complementares, racionalidades médicas e promoção da saúde: contribuições poucos exploradas. Cadernos de Saúde Pública, 25(8), 1732-1742. http://dx.doi.org/10.1590/S0102-311X2009000800009

Whittemore R, \& Knafl K. (2005). The integrative review: updated methodology. Journal of Advanced Nursing, 52(5), 546-553. http://dx.doi.org/10.1111/j.1365- 2648.2005.03621.x

World Health Organization (WHO). (2020). Coronavirus disease (COVID-19) outbreak situation. Recuperado em mai 30, 2020 de https://www.who.int/emergencies/diseases/novelcoronavirus-2019

World Health Organization (WHO). (2002). Tradicional Medicine Strategy:2014:2023. http://www.wpro.who.int/health_ technology/book_who_traditional_medicine_strategy_ 2002_2005.pdf 